Research Article

\title{
Natural Hydroxyapatite: Green Catalyst for the Synthesis of Pyrroles, Inhibitors of Corrosion
}

\author{
Abdelhadi Louroubi $\left(\mathbb{D},{ }^{1}\right.$ Abdallah Nayad $(D),{ }^{1}$ Ali Hasnaoui ${ }^{D},{ }^{1}$ Rachid Idouhli $(D), 2$ \\ Abdessalam Abouelfida $\mathbb{D}^{D},{ }^{2}$ Larbi El Firdoussi $\mathbb{D}^{1},{ }^{1}$ and Mustapha Ait Ali $\mathbb{D}^{1}$ \\ ${ }^{1}$ Molecular Chemistry Laboratory, Coordination Chemistry and Catalyse Unit, Faculty of Sciences Semlalia, \\ Cadi Ayyad University, Marrakech, Morocco \\ ${ }^{2}$ Physical-Chemistry of Materials and Environment Laboratory, Faculty of Sciences Semlalia, Cadi Ayyad University, \\ Marrakech, Morocco \\ Correspondence should be addressed to Mustapha Ait Ali; aitali@uca.ac.ma
}

Received 29 November 2020; Revised 15 February 2021; Accepted 24 February 2021; Published 5 March 2021

Academic Editor: Liviu Mitu

Copyright (C) 2021 Abdelhadi Louroubi et al. This is an open access article distributed under the Creative Commons Attribution License, which permits unrestricted use, distribution, and reproduction in any medium, provided the original work is properly cited.

\begin{abstract}
Polysubstituted pyrroles have been synthesized in good yields via a four-component one-pot reaction of 1,3-dicarbonyl compounds, amines, aldehydes, and nitroalkanes using natural hydroxyapatite (HAp) as an efficient green catalyst. This strategy provides advantages such as simple experimental and work-up procedures, mild conditions, high selectivity, low cost, high atom economy, and environmental friendliness; it uses a green commercial catalyst and does not require a solvent. The electrochemical behavior of S300 steel in $1 \mathrm{M}$ hydrochloric acidic was studied in the presence of these heterocyclic compounds. The results showed good inhibition efficiency for steel in acidic media.
\end{abstract}

\section{Introduction}

Pyrrole derivatives are an important class of heterocyclic compounds found in a large variety of natural products and important biological molecules [1] and exhibit interesting bioactivity proprieties [2]. Therefore, many methods have been developed to synthesize pyrrole derivatives [3-5]. Among them, multicomponent reaction (MCR) coupling, a procedure allowing the synthesize of a target molecule from three or more starting materials, has emerged as a popular and core strategy to construct functionalized pyrrole derivatives, which have been extensively reviewed with different starting materials [6]. MCRs provide interesting advantages, including the synthesis of high functionalized molecules in one pot with good overall yields without the need for the isolation and purification of the intermediate product; it has high selectivity and operational simplicity and, hence, minimal waste, time, labor, and cost [7]. Thus, this straightforward approach could be an environmentally friendly and economical alternative to modern organic synthesis and combinatorial and medicinal chemistry [8]. Recently, a new four-component reaction employing 1,3dicarbonyl compounds, amines, aldehydes, and nitroalkanes to synthesize tetra-substituted pyrroles derivatives has been performed using $\mathrm{FeCl}_{3}$ [9], iodine [10], $\mathrm{NiCl}_{2} \cdot 6 \mathrm{H}_{2} \mathrm{O}$ [11], $[\mathrm{HBim}] \mathrm{BF}_{4}$ [12], and silica tungsten acid (STA) [13]. However, these methods use toxic metals as homogeneous catalysts which are not recyclable, and although ionic liquids are considered as a mild and environmentally friendly solvent in modern organic chemistry [14], the presence of impurities can greatly affect their physicochemical properties [15], and their toxicities are not well known [16].

Nowadays, one of the main goals of modern organic synthetic chemistry is the search for environmentally friendly processes and methods. This new age of synthesis requires the development of clean and safe processes, less expensive resources, and lower energy requirements. In this context, heterogeneous catalysts are gaining popularity in green chemistry protocols. Only a few recent studies have reported using heterogeneous catalysts in the synthesis of 
pyrrole derivatives with an MCR approach [17-19]. However, the catalysts were not readily available or contained expensive starting materials. To address this problem, the development of a green protocol using a commercially cheap heterogeneous catalyst for the synthesis of functionalized pyrroles is highly desired, especially if the catalyst can be recovered at the end of the reaction without trace amounts of metal contamination [20].

Corrosion is a costly problem for the industry field [21]. Therefore, many studies have been done to determine how to prevent metallic aggression against air and water pollution or how to remove rust or any undesirable deposits on the surface of metals caused by chemical or/and electrochemical reactions. One of the most attractive methods is the utilization of synthetic organic compounds as corrosion inhibitors [22]. Heterocyclic compounds containing donor atoms such as $\mathrm{N}, \mathrm{O}, \mathrm{S}$, and $\mathrm{P}$, as well as unsaturated bonds (electron pairs or/and $\pi$-electron), and polar functional groups (e.g., $-\mathrm{OCH}_{3},-\mathrm{CH}_{3},-\mathrm{NH}_{2}$, and $-\mathrm{Cl}$ ) were identified as the most effective inhibitors and are widely reviewed in the literature [23]. They are adsorbed onto the metal surface and prevent metal dissolution in aggressive media [24]. Lately, we investigated the corrosion inhibition of S300 steel in $1 \mathrm{M} \mathrm{HCl}$ using a pyrrole derivative [25].

We have recently reported a simple, rapid, and efficient synthesis of $\beta$-ketoester using $\mathrm{HAp}\left(\mathrm{Ca}_{5}\left(\mathrm{PO}_{4}\right)_{3} \mathrm{OH}\right)$ as a catalyst [26]. The catalyst used in this work is a bioceramic, which can be synthesized by various methods such as hydrothermal, sol-gel, precipitation, and emulsion [27]. Despite good thermal and chemical stability, bioactivity, adsorption capacity, macroligant behavior, and acid-base propriety [28], the potential of HAp applications in catalysis support in the synthesis of polyheterocyclic compounds has been less explored [29]. In our efforts for the development of ecocompatible procedures, we report here a convenient synthesis of polysubstituted pyrroles via a four-component condensation reaction catalyzed by natural HAp in mild conditions. The corrosion inhibition of different prepared compounds was also performed using the potentiodynamic polarization method.

\section{Experimental Section}

2.1. Materials. NMR studies were performed on a Bruker Avance 300 spectrometer in $\mathrm{CDCl}_{3}$; chemical shifts are given in $\mathrm{ppm}$ relative to external TMS. The reaction mixtures were analyzed on a Trace GC Shimadzu chromatograph equipped with an FID detector. GC parameters for capillary columns TG-5MS $(30 \mathrm{~m} \times 0.25 \mathrm{~mm})$ are as follows: injector, $230^{\circ} \mathrm{C}$; detector, $200^{\circ} \mathrm{C}$; oven, $40^{\circ} \mathrm{C}$ for $2 \mathrm{~min}$, followed by $40^{\circ} \mathrm{C} \mathrm{min}{ }^{-1}$ until $280^{\circ} \mathrm{C}$ for $12 \mathrm{~min}$; column pressure, $42.9 \mathrm{kPa}$; gas flow, $20 \mathrm{~mL} \mathrm{~min}^{-1}$. The spectrum mass of the products was obtained by ionization on an ISQ LT single quadruple mass spectrometer in positive EI mode using a mass scan range of 50 to 400 da. Liquid chromatography was performed on silica gel (Merck 60, 220-440 mesh). All reagents and solvents used in the experiments were purchased from commercial sources and used without further purification (Aldrich, Acros).
2.2. General Procedure for the Synthesis of Pyrrole. To a stirred mixture of amine $(2.5 \mathrm{mmol})$, aldehyde $(2.5 \mathrm{mmol}), 1,3$ dicarbonyl (2.5 mmol), and nitroalkane ( $5 \mathrm{mmol}$ ), HAp (S/ $\mathrm{C}=60$ ) was added as a catalyst. The reaction was carried out at $60^{\circ} \mathrm{C}$ for $24 \mathrm{~h}$. After completion of the reaction, the resultant crude product was cooled to room temperature, then diluted with water $(5 \mathrm{~mL})$, and extracted with ethyl acetate ( 3 ч $5 \mathrm{~mL}$ ). The organic residue was dried over $\mathrm{Na}_{2} \mathrm{SO}_{4}$, and the solvent was removed under reduced pressure. The residue was purified by column chromatography over silica gel (Merck 60, 220-440 mesh) using a mixture of hexane/ethylacetate $(9.5 / 0.5: \mathrm{v} / \mathrm{v})$ as an eluent to get the pyrrole desired.

2.3. Synthesis of 4-(Benzylamino)pent-3-en-2-one. By following our reported procedure [26], a mixture of $1.7 \mathrm{mmol}$ of acetylacetone, $1.7 \mathrm{mmol}$ of benzylamine, and $0.017 \mathrm{mmol}$ of $\mathrm{Ca}_{5}\left(\mathrm{PO}_{4}\right)_{3} \mathrm{OH}$ were stirred at room temperature for $10 \mathrm{~min}$. After completion of the reaction, $10 \mathrm{~mL}$ of distilled water was added, and the product was extracted by ethyl acetate $(3 \times 25 \mathrm{ml})$. The organic layer was dried with $\mathrm{Na}_{2} \mathrm{SO}_{4}$, and the solvent was eliminated under vacuum. The desired product was obtained by silica gel column chromatography by using a mixture of hexane/ethylacetate (96/4: v/v) as an eluent.

2.4. Electrochemical Measurements. Electrochemical tests were performed using a potentiostat PGZ100 instrument piloted by VoltaMaster 4 software. The instrument was attached to a jacketed glass cell $\left(20 \pm 1^{\circ} \mathrm{C}\right)$ with a threeelectrode configuration. A platinum sheet $\left(2 \mathrm{~cm}^{2}\right)$ was used as the auxiliary electrode, silver-silver chloride $(\mathrm{Ag} / \mathrm{AgCl})$ was selected as the reference electrode, and $0.76 \mathrm{~cm}^{2}$ of $\mathrm{S} 300$ steel was used as the working electrode with a chemical composition of Fe (98.55\%), C (0.15\%), Mn (1.25\%), and Si $(0.05 \%)$. Prior to each experiment, the working electrode was polished with various grades of sand paper, washed, and immersed in an acidic solution. The electrochemical measurements were carried out after $30 \mathrm{~min}$ of immersion time to establish the open circuit potential (OCP). The polarization curves were studied in the potential range of $-0.8 \mathrm{~V}$ to $-0.2 \mathrm{~V}$ at a scanning rate of up to $1 \mathrm{mV} / \mathrm{sec}$.

The inhibition efficiency $(\eta \%)$ was calculated using the following relation:

$$
\eta(\%)=\frac{i_{\text {corr }}^{\prime}-i_{\text {corr }}}{i_{\text {corr }}^{\prime}} \times 100,
$$

where $i_{\text {corr }}$ and $i_{\text {corr }}^{\prime}$ are the corrosion current density without and with inhibitor.

\section{Results and Discussion}

3.1. The Four Synthesis Routes of the Pyrrole Derivatives. Initially, we were interested in the synthesis of a pyrrole moiety catalyzed by HAp by four methods (Scheme 1). For this reason, we examined the four-component reaction of acetylacetone $(2.5 \mathrm{mmol})$, benzylamine $(2.5 \mathrm{mmol})$, benzaldehyde $(2.5 \mathrm{mmol})$, and nitromethane $(5 \mathrm{mmol})$ (Method 

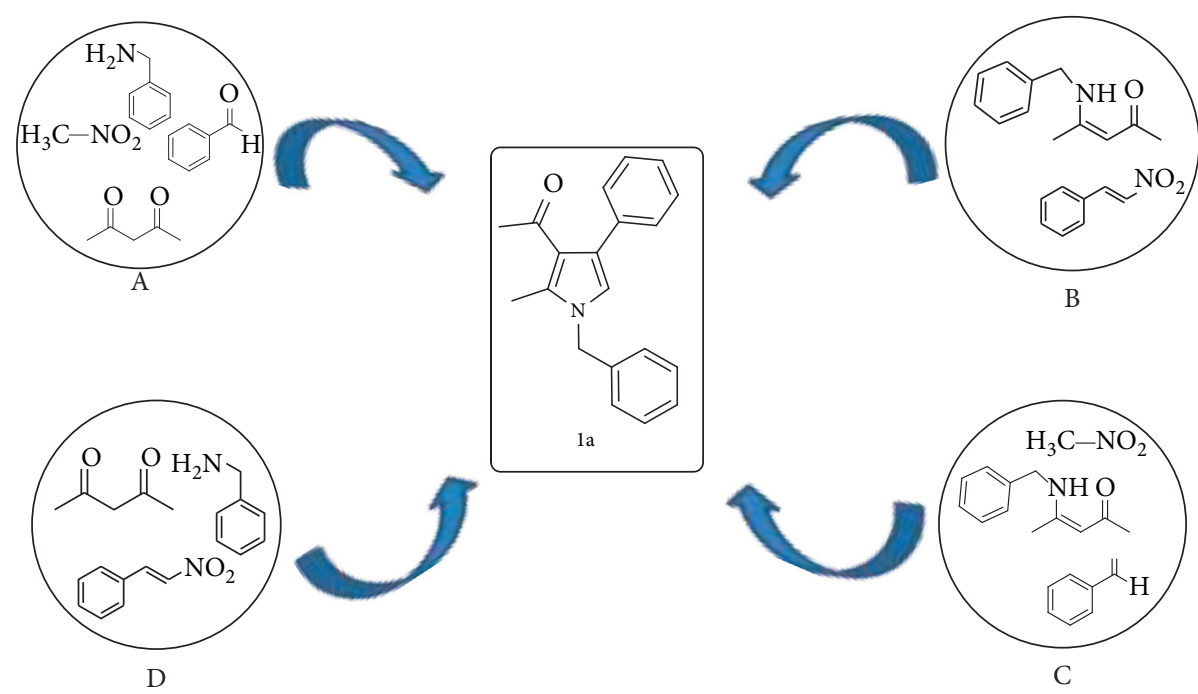

Scheme 1: Synthesis routes of pyrrole derivatives.

A). The three-component reaction involved 4-(benzylamino) pent-3-en-2-one $(2.5 \mathrm{mmol})$, benzaldehyde $(2.5 \mathrm{mmol})$, and nitromethane $(5 \mathrm{mmol})$ (Method $\mathrm{C}$ ) or $\beta$-nitrostyrene $(2.5 \mathrm{mmol})$, acetylacetone $(2.5 \mathrm{mmol})$, and benzylamine (2.5 mmol) (Method D). Method B used two compounds in the reaction: 4-(ben9zylamino)pent-3-en-2-one $(2.5 \mathrm{mmol})$ and $\beta$-nitrostyrene $(2.5 \mathrm{mmol})$. The yields of product $1 \mathrm{a}$ are given in Table 1.

We found that the one-pot synthesis (Method A) gave a better yield of the desired pyrrole 1a with a total conversion (Table 1, entry 1). Similar results for the synthesis of 1a were described with other catalysts $[11,19]$. Method B (Table 1, entry 2) did not provide a good yield, and we noticed that nitrostyrene was less reactive in the process of the reaction. The same was observed for the three-component methods (Table 1, entries 3 and 4). Our attention was then turned to the four-component reactions described in Method A. We chose the reaction of acetylacetone $(2.5 \mathrm{mmol})$, methyl benzylamine $(2.5 \mathrm{mmol})$, benzaldehyde $(2.5 \mathrm{mmol})$, and nitromethane $(5 \mathrm{mmol})$ as the standard reaction (Scheme 2) to find the optimal conditions to improve the yield. For this purpose, various amounts of the catalyst and different solvents and temperatures were screened (Table 2).

3.2. Optimization of the Four-Component Reaction. The experiment performed without the catalyst in solvent-free condition at $60^{\circ} \mathrm{C}$ was less effective; only $20 \%$ of the product $1 \mathrm{~b}$ was isolated (Table 2, entry 1). We then examined the effect of HAp loading in the solvent-free reaction. The results indicated that, for a molar ratio of 60 , the reaction led to a good yield of $89 \%$ (Table 2, entry 3 ). The use of the catalyst is, therefore, crucial for the evolution of the reaction. However, we observed that an increase in the amount of the catalyst decreased the yield of the desired product (Table 2, entries 4 and 5).

We studied different solvents in our standard reaction using HAp $(\mathrm{S} / \mathrm{C}=60)$ as the catalyst at $60^{\circ} \mathrm{C}$. The use of polar solvents, such as water and ethanol, gave good yields of the desired product (Table 2, entries 6 and 7), as opposed to acetonitrile, which only gave a yield of $6 \%$ (Table 2 , entry 8 ). It can be explained by the protic character of water and ethanol which participates in the reaction medium by hydrogen bonding. In the case of toluene, only traces of the product were found under the reaction conditions (Table 2, entry 9). The best protocol for this reaction was solvent free (Table 2, entry 3 ) because it allows a direct interaction of all starting materials. We also studied the effect of temperature on the catalytic reaction. At room temperature (RT) and $40^{\circ} \mathrm{C}$, only 21 and $24 \%$ of the products were isolated, respectively (Table 2, entries 10 and 11). However, the yield of the product decreased when the temperature of the reaction increased to $90^{\circ} \mathrm{C}$ and $120^{\circ} \mathrm{C}$ (Table 2, entries 12 and 13 ). The result shows that the best thermodynamic condition to perform the reaction was at a temperature of $60^{\circ} \mathrm{C}$ that afforded the highest yield towards the product $1 \mathrm{~b}$. To define a more efficient catalytic system for the synthesis of pyrrolic products, we have performed the reaction with other types of phosphate-based catalysts such as $\mathrm{AlPO}_{4}$ and fluoroapatite (FAP) (Table 2 ). We found that both catalysts afforded the same yield (Table 2, entries 14 and 15). However, the presence of natural HAp in the four-component reaction gave the best results due to a better interaction of hydroxyl groups from HAp catalyst with the starting materials.

3.3. Kinetic Study of the Four-Component Reaction. The evolution of the reaction through the time was studied under the optimized conditions (Figure 1). Every two hours, a sample is taken from the reaction medium and then extracted with ethyl acetate and analyzed by GC in order to determine the selectivity of the obtained products. Three main products were detected: two intermediate products-PI1: (4-phenylamino-pent-3-en-2-one) and PI2: nitrostyrene-and the corresponding pyrrole derivative (Scheme 3). They were isolated and confirmed by RMN study. 
TABLE 1: Preparation of $1 \mathrm{a}^{\mathrm{a}}$ by the four synthesis routes.

\begin{tabular}{|c|c|c|c|c|}
\hline Entry & Methods & Reagents & & Yield $^{\mathrm{b}}(\%)$ \\
\hline 1 & A & & $\mathrm{H}_{3} \mathrm{C} \longrightarrow \mathrm{NO}_{2}$ & 74 \\
\hline 2 & B & & & 39 \\
\hline 3 & $\mathrm{C}$ & & $\mathrm{H}_{3} \mathrm{C}-\mathrm{NO}_{2}$ & 36 \\
\hline 4 & $\mathrm{D}$ & & & 10 \\
\hline
\end{tabular}

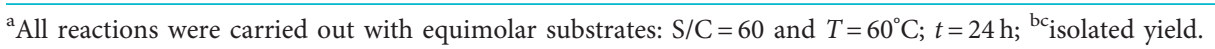

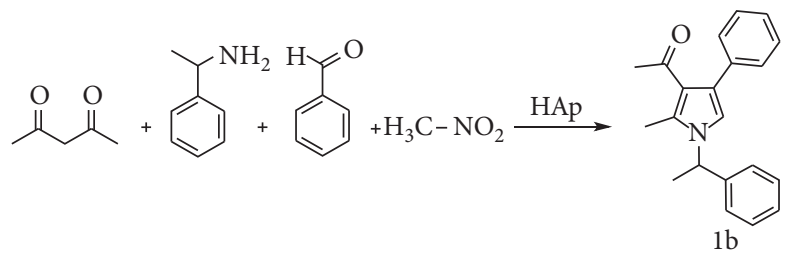

Scheme 2: The standard reaction for optimization.

TABLE 2: Optimization of the standard reaction ${ }^{\mathrm{a}}$.

\begin{tabular}{|c|c|c|c|c|}
\hline Entry & Solvent & $\mathrm{T}\left({ }^{\circ} \mathrm{C}\right)$ & Molar ratio (S/C) & Yield $^{\mathrm{b}}(\%)$ \\
\hline 1 & Free solvent & 60 & 0 & 20 \\
\hline 2 & Free solvent & 60 & 100 & 68 \\
\hline 3 & Free solvent & 60 & 60 & 89 \\
\hline 4 & Free solvent & 60 & 40 & 63 \\
\hline 5 & Free solvent & 60 & 20 & 25 \\
\hline 6 & $\mathrm{H}_{2} \mathrm{O}$ & 60 & 60 & 80 \\
\hline 7 & Ethanol & 60 & 60 & 78 \\
\hline 8 & Acetonitrile & 60 & 60 & 6 \\
\hline 9 & Toluene & 60 & 60 & Trace \\
\hline 10 & Free solvent & RT & 60 & 21 \\
\hline 11 & Free solvent & 40 & 60 & 24 \\
\hline 12 & Free solvent & 90 & 60 & 75 \\
\hline 13 & Free solvent & 120 & 60 & 55 \\
\hline 14 & Free solvent & 60 & 60 & $78^{\mathrm{c}}$ \\
\hline 15 & Free solvent & 60 & 60 & $77^{\mathrm{d}}$ \\
\hline
\end{tabular}

${ }^{\mathrm{a}}$ All reactions were carried out using aldehyde $(2.5 \mathrm{mmol})$, amine $(2.5 \mathrm{mmol})$, and 1,3-dicarbonyl compound $(2.5 \mathrm{mmol})$ to nitromethane $(5 \mathrm{mmol}), t=24 \mathrm{~h}$.

bisolated yield. ${ }^{\mathrm{c}}$ Reaction with $\mathrm{AlPO}_{4}$ as the catalyst. ${ }^{\mathrm{d}}$ Reaction was conducted in the presence of fluoro-apatite (FAP) as the catalyst. 


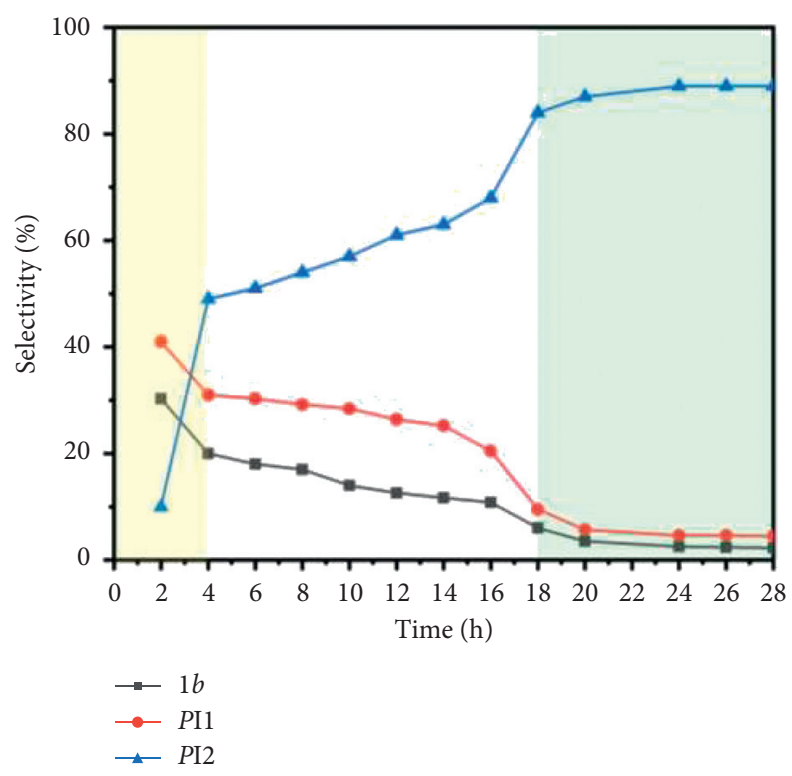

FIGURE 1: Evolution of the selectivity of the intermediate products (PI1 and PI2) and the pyrrolic product $1 \mathrm{~b}$ as a function of time.

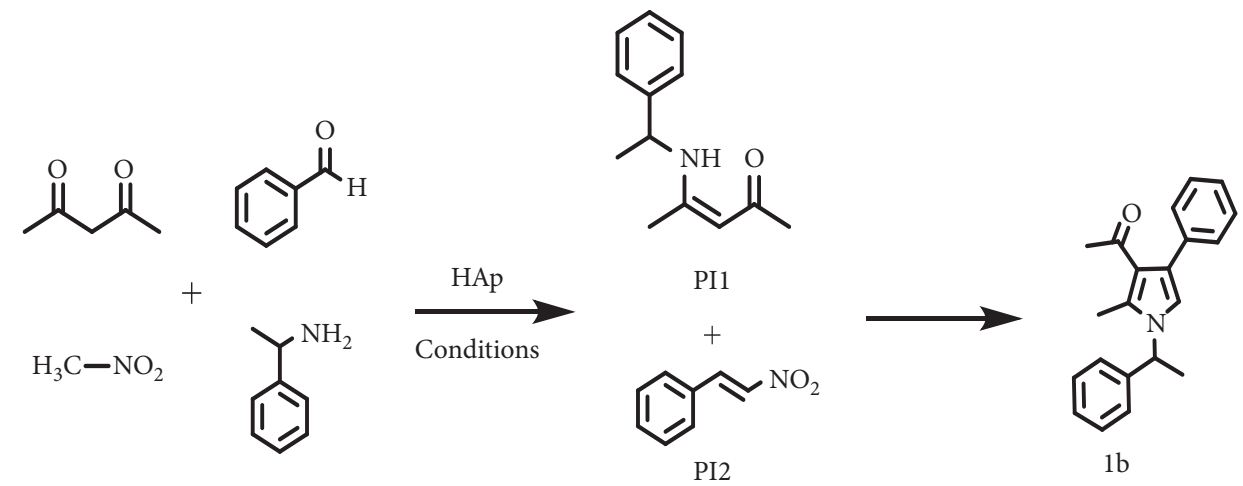

Scheme 3: Reaction of pyrrole synthesis.

As illustrated in Figure 1, the evolution of the reaction towards the formation of the pyrrole derivative goes through intermediates (PI1 and PI2). According to the curve below, at $t=2 \mathrm{~h}$, the pyrrole $1 \mathrm{~b}$ is formed in a very small amount (10\%), while the intermediates PI1 and PI2 are found as the main formed products in the reaction medium with $41 \%$ and $30 \%$, respectively.

After $4 \mathrm{~h}$, the reaction showed an increase in the selectivity towards the product $1 \mathrm{~b}(49 \%)$, which resulted in the decrease in the amount of the PI1 and PI2 in the reaction medium. The reaction started to reach stabilization after $18 \mathrm{~h}$, with a maximum isolated yield of $89 \%$.

3.4. Multicomponent Synthesis of Pyrroles by Various Substrates. After determining the optimal reaction conditions, we extended the experimental protocol by studying the scope and limitations of this straightforward fourcomponent reaction (Scheme 4), and the results are summarized in Table 3. We first ran the reaction with acetylacetone, benzaldehyde, and nitroethane with different aromatic and aliphatic amines. The 1-phenylethylamine resulted in an excellent yield (90\%) of product 1d (Table 3, entry 2), and benzylamine generated the corresponding pyrrole 1e with a yield of $74 \%$ (Table 3 , entry 3 ). Aniline and isopropylamine exhibited moderated yields of $48 \%$ and $44 \%$, respectively (Table 3 , entries 1 and 4 ). Thus, better yields were obtained with aliphatic amines bearing an aromatic radical rather than aromatic amines and simple aliphatic amines. Encouraged by these results, we expanded the fourcomponent procedure by examining the effect of different aromatic aldehydes. The pyrrolic products prepared from $p$ chlorobenzaldehyde and $p$-methylbenzaldehyde were isolated with good yields of $67 \%$ and $77 \%$, respectively (Table 3 , entries 6 and 10). This observation showed that the yield was affected by weak electron-donating and strong electronwithdrawing substituents of aromatic aldehydes.

Furthermore, this four-component method is suitable for both nitromethane and nitroethane, giving the corresponding pyrrolic products similar yields. These results prove that this 


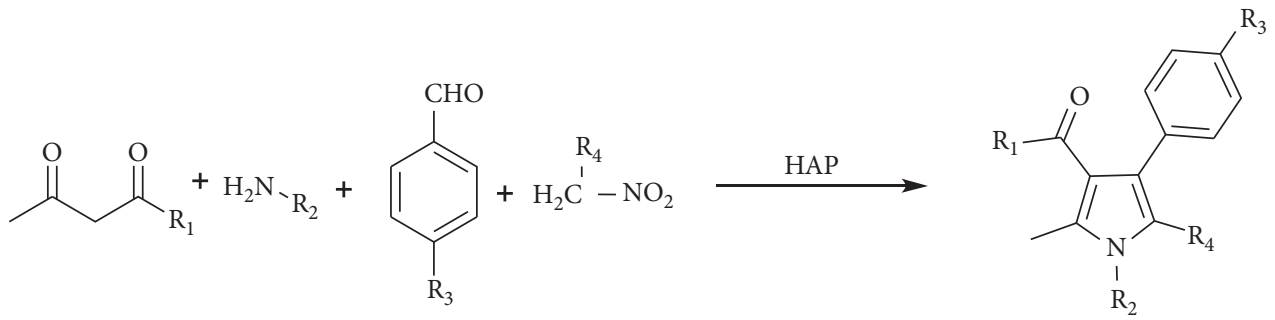

Scheme 4: Multicomponent synthesis of pyrroles by various substrates.

TABLE 3: Multicomponent synthesis of pyrroles by various substrates ${ }^{\mathrm{a}}$.

\begin{tabular}{|c|c|c|c|c|c|c|}
\hline Entry & $R_{1}$ & $R_{2}$ & $R_{3}$ & $R_{4}$ & Products & Yields $^{\mathrm{b}}(\%)$ \\
\hline 1 & $\mathrm{Me}$ & $\mathrm{Ph}$ & $\mathrm{H}$ & $\mathrm{Me}$ & $1 c$ & 48 [30] \\
\hline 2 & $\mathrm{Me}$ & $\mathrm{CH}_{3} \mathrm{CH}(\mathrm{Ph})$ & $\mathrm{H}$ & $\mathrm{Me}$ & $1 \mathrm{~d}$ & 90 \\
\hline 3 & $\mathrm{Me}$ & $\mathrm{Ph}-\mathrm{CH}_{2}$ & $\mathrm{H}$ & $\mathrm{Me}$ & le & $74[31]$ \\
\hline 4 & $\mathrm{Me}$ & $\mathrm{CH}_{3} \mathrm{CHCH}_{3}$ & $\mathrm{H}$ & $\mathrm{Me}$ & 1f & 44 \\
\hline 5 & $\mathrm{Me}$ & $\mathrm{Ph}$ & $\mathrm{Cl}$ & $\mathrm{Me}$ & $1 \mathrm{~g}$ & 50 \\
\hline 6 & $\mathrm{Me}$ & $\mathrm{Ph}-\mathrm{CH}_{2}$ & $\mathrm{Cl}$ & $\mathrm{Me}$ & $1 \mathrm{~h}$ & $67[25]$ \\
\hline 7 & $\mathrm{Me}$ & $\mathrm{CH}_{3} \mathrm{CH}(\mathrm{Ph})$ & $\mathrm{Cl}$ & $\mathrm{Me}$ & $1 \mathrm{i}$ & 44 \\
\hline 8 & $\mathrm{Me}$ & $\mathrm{CH}_{3} \mathrm{CHCH}_{3}$ & $\mathrm{Cl}$ & $\mathrm{Me}$ & $1 \mathrm{j}$ & 41 \\
\hline 9 & $\mathrm{Me}$ & $\mathrm{Ph}$ & $\mathrm{Me}$ & $\mathrm{Me}$ & $1 \mathrm{k}$ & 63 \\
\hline 10 & $\mathrm{Me}$ & $\mathrm{Ph}-\mathrm{CH}_{2}$ & $\mathrm{Me}$ & $\mathrm{Me}$ & 11 & 77 \\
\hline 11 & $\mathrm{Me}$ & $\mathrm{CH}_{3} \mathrm{CH}(\mathrm{Ph})$ & $\mathrm{Me}$ & $\mathrm{Me}$ & $1 \mathrm{~m}$ & 64 \\
\hline 12 & $\mathrm{Me}$ & $\mathrm{Ph}-\mathrm{CH}_{2}$ & 1-Naphthyl & $\mathrm{Me}$ & ln & 37 \\
\hline 13 & $\mathrm{Me}$ & $\mathrm{CH}_{3} \mathrm{CH}(\mathrm{Ph})$ & 1-Naphthyl & $\mathrm{Me}$ & 10 & 39 \\
\hline 14 & $\mathrm{Me}$ & $\mathrm{CH}_{3} \mathrm{CHCH}_{3}$ & 1-Naphthyl & $\mathrm{Me}$ & $1 \mathrm{p}$ & 90 \\
\hline 15 & OEt & $\mathrm{Ph}-\mathrm{CH}_{2}$ & $\mathrm{Me}$ & $\mathrm{H}$ & $1 \mathrm{q}$ & 29 \\
\hline 16 & OEt & $\mathrm{Ph}$ & $\mathrm{H}$ & $\mathrm{H}$ & $1 \mathrm{r}$ & 30 \\
\hline 17 & OEt & $\mathrm{Ph}-\mathrm{CH}_{2}$ & $\mathrm{H}$ & $\mathrm{H}$ & $1 \mathrm{~s}$ & 43 \\
\hline 18 & OEt & $\mathrm{CH}_{3} \mathrm{CH}(\mathrm{Ph})$ & $\mathrm{H}$ & $\mathrm{H}$ & $1 \mathrm{t}$ & 41 \\
\hline 19 & OEt & $\mathrm{CH}_{3} \mathrm{CHCH}_{3}$ & $\mathrm{H}$ & $\mathrm{H}$ & $1 \mathrm{u}$ & 29 \\
\hline
\end{tabular}

${ }^{\text {a}}$ Reactions were carried out using aldehyde $(2.5 \mathrm{mmol})$, amine $(2.5 \mathrm{mmol})$, and 1,3-dicarbonyl compounds (2.5 mmol) to nitromethane (5 mmol), HAp (S/ C) $=60, T=60^{\circ} \mathrm{C}, t=24 \mathrm{~h}$. ${ }^{\mathrm{b}}$ Isolated yield.

method is extendable to a variety of nitroalkane compounds. The structures of products $1 \mathrm{e}$ and $1 \mathrm{~h}$ were successfully confirmed by single-crystal X-ray crystallographic analysis $[25,31]$.

We have also investigated the effect of another 1,3dicarbonyl compound named ethylacetoacetate, on benzaldehyde, nitromethane, and diverse amines such as 1-phenylethanamine, benzylamine, aniline, and isopropylamine. The corresponding products were obtained in $43 \%, 41 \%, 30 \%$, and $29 \%$ yields, respectively (Table 3, entries 17-21). Compared to acetoacetylacetone, this four-component coupling reaction was found to be less reactive with $\beta$-ketoester compounds. $\mathrm{RMN}$ and mass spectra of products $1 \mathrm{k}, 1 \mathrm{~m}$, and $1 \mathrm{p}$ can be found in supplementary data.

Based on the literature $[9,10]$, we have proposed a plausible mechanism for the one-pot reaction (Scheme 5). The intermediate products $\beta$-nitrostyrene and $\beta$-enamino ketone were both separated and isolated in the reaction process. This corroborates with the research of Grob and Camenisch that pyrroles can be obtained from a Michael reaction of $\beta$-enamino ketones or esters and nitroalkenes followed by cyclization [32].
The synthesis of fully functionalized pyrrole derivatives by this four-component reaction was less explored in the literature. Table 4 summarizes the comparison of our work with other reported catalysts.

3.5. Results of Mass Spectrometry and NMR Spectra. 1-(1-Benzyl-5-methyl-3-phenyl-2H-pyrrole)ethanone (1a). Orange sticky liquid (535 mg, 74\%); ${ }^{1} \mathrm{H}$ NMR $\left(\mathrm{CDCl}_{3}\right.$, $300 \mathrm{MHz}) \delta=1.94\left(\mathrm{~s}, 3 \mathrm{H} ; \mathrm{CH}_{3}\right), 2.33\left(\mathrm{~s}, 3 \mathrm{H} ; \mathrm{CH}_{3}\right), 4.93$ (s, $2 \mathrm{H} ; \mathrm{CH}_{2}$ ), 6.42 (s, 1H; CH), 6.96-7.23 ppm (m, 10H; Ar-H); ${ }^{13} \mathrm{C} \mathrm{NMR}\left(\mathrm{CDCl}_{3}, 75 \mathrm{MHz}\right) \delta=11.6\left(\mathrm{~s} ; \mathrm{CH}_{3}\right), 31.1\left(\mathrm{~s} ; \mathrm{CH}_{3}\right)$, $50.3\left(\mathrm{~s} ; \mathrm{CH}_{2}\right), 120.2(\mathrm{C}=\mathrm{CH}), 125.9$ (CC), 126.7 (CC), 126.7 (CHar), 127.9 (CHar), 128.2 (CHar), 1289.0 (CHar), 129.4 (CHar), 135.2 (CC), 136.3 (CC), 136.6 (CC), and 197.6 $(\mathrm{C}=\mathrm{O})$.

1-(2-Methyl-4-phenyl-1-(1-phenylethyl)-1H-pyrrol-3yl)ethanone (1b). Solid orange $(576 \mathrm{mg}, 76 \%) ;{ }^{1} \mathrm{H}$ NMR $\left(\mathrm{CDCl}_{3}, 300 \mathrm{MHz}\right) \delta=1.70\left(\mathrm{~d}, 3 \mathrm{H} ; \mathrm{CH}_{3}\right), 1.93\left(\mathrm{~s}, 3 \mathrm{H} ; \mathrm{CH}_{3}\right)$, 2.31 (s, 3H; $\left.\mathrm{CH}_{3}\right), 5.26(\mathrm{q}, 1 \mathrm{H} ; \mathrm{CH}), 6.57$ (s, 1H; Ar-H), 6.97-7.24 ppm (m, 10H; $\mathrm{Ar}-\mathrm{H}) ;{ }^{13} \mathrm{C} \mathrm{NMR}\left(\mathrm{CDCl}_{3}, 75 \mathrm{MHz}\right)$ $\delta=11.5\left(\mathrm{~s} ; \mathrm{CH}_{3}\right), 22.2\left(\mathrm{~s} ; \mathrm{CH}_{3}\right), 31.2\left(\mathrm{~s} ; \mathrm{CH}_{3}\right), 54.9(\mathrm{~s} ; \mathrm{CH})$, $116.6(\mathrm{C}=\mathrm{CH}), 122.0$ (CC), 125.7 (CC), 125.9 (CHar), 126.6 


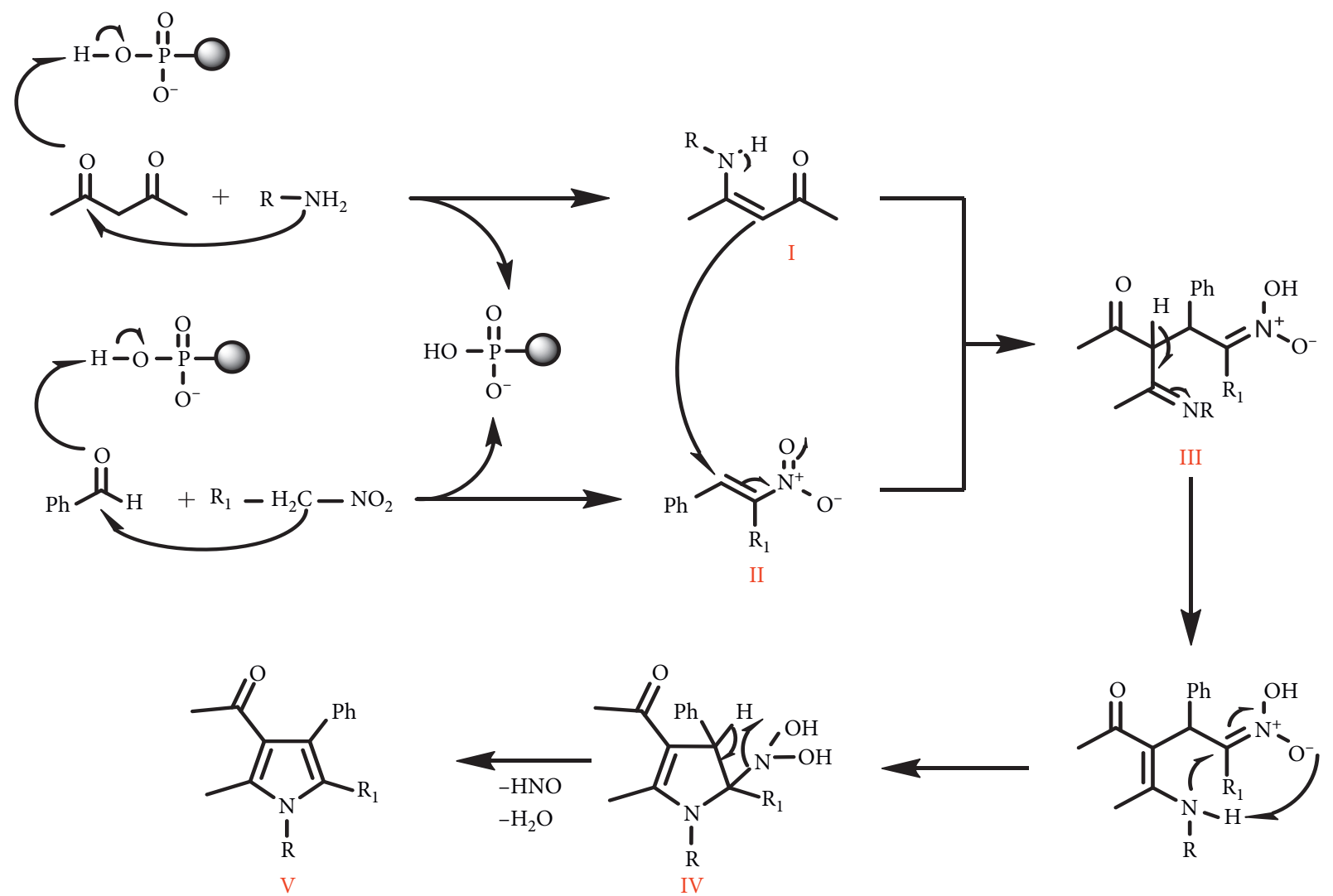

Scheme 5: The mechanism proposed for the synthesis of pyrroles.

TABLE 4: Comparison of HAp with other catalysts for the synthesis of fully functionalized pyrrole derivatives.

\begin{tabular}{lcccr}
\hline Entry & Catalyst & Temperatures & Yield (\%) & Reference \\
\hline 1 & $\mathrm{NiCl}_{2} \cdot 6 \mathrm{H}_{2} \mathrm{O}$ & Reflux & 60 & {$[11]$} \\
2 & $\mathrm{STA}$ & Reflux & 88 & {$[13]$} \\
3 & $\mathrm{HAp}$ & $60^{\circ} \mathrm{C}$ & 90 & This work \\
\hline
\end{tabular}

(CHar), 127.7 (CHar), 128.3 (CHar), 128.9 (CHar), 129.4 (CHar), 135.1 (CC), 136.6 (CC), 142.0 (CC), 197.9 (C=O).

1-(2,5-Dimethyl-1,4-diphenyl-1H-pyrrol-3-yl)ethanone (1c). Yellow solid (347 mg, 48\%); ${ }^{1} \mathrm{H}$ NMR (DMSO, $300 \mathrm{MHz}) \delta=1.79\left(\mathrm{~s}, 3 \mathrm{H} ; \mathrm{CH}_{3}\right), 1.84\left(\mathrm{~s}, 3 \mathrm{H} ; \mathrm{CH}_{3}\right), 2.18(\mathrm{~s}$, $\left.3 \mathrm{H} ; \mathrm{CH}_{3}\right), \quad 7.26-7.58 \mathrm{ppm}(\mathrm{m}, 10 \mathrm{H} ; \mathrm{Ar}-\mathrm{H}) ;{ }^{13} \mathrm{C} \mathrm{NMR}$ (DMSO, $75 \mathrm{MHz}) \delta=10.9\left(\mathrm{~s} ; \mathrm{CH}_{3}\right), 12.7\left(\mathrm{~s} ; \mathrm{CH}_{3}\right), 30.6(\mathrm{~s}$; $\mathrm{CH}_{3}$ ), 121.1 (CC), 121.3 (CC), 126.1 (CHar), 126.4 (CC), 128.1 (CHar), 128.2 (CHar), 129.6 (CHar), 130.2 (CHar), 133.7 (CC), 136.3 (CC), 136.8 (CC), 195.1 ppm (C=O); ESIMS (positive mode) $m / z=289.1[\mathrm{M}]^{+}$.

1-(2,5-Dimethyl-4-phenyl-1-(1-phenylethyl)-1H-pyrrol-3-yl)ethanone (1d). Brown sticky liquid (713 mg, 90\%); ${ }^{1} \mathrm{H}$ NMR $\left(\mathrm{CDCl}_{3}, 300 \mathrm{MHz}\right) \delta=1.71\left(\mathrm{~d}, 3 \mathrm{H} ; \mathrm{CH}_{3}\right), 1.78(\mathrm{~s}$, $3 \mathrm{H} ; \mathrm{CH}_{3}$ ), 1.95 (s, 3H; $\mathrm{CH}_{3}$ ), 2.33 (s, 3H; $\mathrm{CH}_{3}$ ), 5.54 (q, $1 \mathrm{H}$; $\mathrm{CH}), 7.02-7.30 \mathrm{ppm}(\mathrm{m}, 10 \mathrm{H}$; $\mathrm{HC}-\mathrm{Ar}) ;{ }^{13} \mathrm{C} \mathrm{NMR}\left(\mathrm{CDCl}_{3}\right.$, $75 \mathrm{MHz}) \delta=11.5\left(\mathrm{~s} ; \mathrm{CH}_{3}\right), 12.6\left(\mathrm{~s} ; \mathrm{CH}_{3}\right), 18.8\left(\mathrm{~s} ; \mathrm{CH}_{3}\right), 31.1$ $\left(\mathrm{s} ; \mathrm{CH}_{3}\right), 52.7$ (s; CH), 122.9 (CC), 125.9 (CC), 126.1 (CHar), 126.5 (CHar), 127.2 (CHar), 128,1 (CHar), 128.4 (CHar), 130.3 (CHar), 130.7 (CHar), 134.0 (CC), 137.3 (CC), 140.9
(CC), 197.7 ppm (C=O); ESI-MS (positive mode) m/ $z=317.1[\mathrm{M}]^{+}$.

1-(1-Benzyl-2,5-dimethyl-4-phenyl-1H-pyrrol-3-yl) ethanone (1e). White solid (561 mg, 74\%); ${ }^{1} \mathrm{H}$ NMR (DMSO, $300 \mathrm{MHz}) \delta=1.76\left(\mathrm{~s}, 3 \mathrm{H} ; \mathrm{CH}_{3}\right), 1.93\left(\mathrm{~s}, 3 \mathrm{H} ; \mathrm{CH}_{3}\right), 2.36(\mathrm{~s}$, $3 \mathrm{H} ; \mathrm{CH}_{3}$ ), 5.18 (s, 2H; $\mathrm{CH}_{2}$ ), 6.97-7.38 ppm (s, 10H; $\left.\mathrm{Ar}-\mathrm{H}\right)$; ${ }^{13} \mathrm{C}$ NMR (DMSO, $\left.75 \mathrm{MHz}\right) \delta=9.9\left(\mathrm{~s} ; \mathrm{CH}_{3}\right), 11.4\left(\mathrm{~s} ; \mathrm{CH}_{3}\right)$, $30.6\left(\mathrm{~s} ; \mathrm{CH}_{3}\right), 46.2\left(\mathrm{~s} ; \mathrm{CH}_{2}\right), 120.7$ (CC), 121.4 (CC), 125.7 (CHar), 125.7 (CHar), 126.4 (CC), 127.1 (CHar), 128.1 (CHar), 128.7 (CHar), 130.2 (CHar), 133.5 (CC), 136.7 (CC), 137.3 (CC), 195,0 ppm (C=O); ESI-MS (positive mode) $\mathrm{m} /$ $z=303.1[\mathrm{M}]^{+}$.

1-(1-Isopropyl-2,5-dimethyl-4-phenyl-1H-pyrrol-3-yl) ethanone (1f). Brown sticky liquid (281 mg, 44\%); ${ }^{1} \mathrm{H}$ NMR $\left(\mathrm{CDCl}_{3}, 300 \mathrm{MHz}\right) \delta=1.54\left(2 \mathrm{~d}, 6 \mathrm{H} ; \mathrm{CH}_{3}\right), 1.82\left(\mathrm{~s}, 3 \mathrm{H} ; \mathrm{CH}_{3}\right)$, 2.12 (s, 3H; $\left.\mathrm{CH}_{3}\right), 2.58$ (s, 3H; $\left.\mathrm{CH}_{3}\right), 4.57$ (m, 1H; $\left.\mathrm{CH}\right)$, 7.38-7.20 ppm (m, 5H; $\mathrm{Ar}-\mathrm{H}) ;{ }^{13} \mathrm{C} \mathrm{NMR}\left(\mathrm{CDCl}_{3}, 75 \mathrm{MHz}\right)$ $\delta=11.7\left(\mathrm{~s} ; \mathrm{CH}_{3}\right), 12.6\left(\mathrm{~s} ; \mathrm{CH}_{3}\right), 21.8\left(\mathrm{~s} ; \mathrm{CH}_{3}\right), 31.04\left(\mathrm{~s} ; \mathrm{CH}_{3}\right)$, $47.4\left(\mathrm{~s} ; \mathrm{CH}_{3}\right.$ ), 121.2 (CC), 122.9 (CC), 125.3 (CC), 126.4 (CHar), 128.1 (CHar), 130.6 (CHar), 133.4 (CC), 137.3 (CC), 
$197.5 \mathrm{ppm}(\mathrm{C}=\mathrm{O})$; ESI-MS (positive mode) $\mathrm{m} / z=255.2$ $[\mathrm{M}]^{+}$.

1-(4-(4-Chlorophenyl)-2,5-dimethyl-1-phenyl-1H-pyrrol3-yl)ethanone (1g). Orange solid (404 mg, 50\%); ${ }^{1} \mathrm{H}$ NMR $\left(\mathrm{CDCl}_{3}, 300 \mathrm{MHz}\right) \delta=1.76\left(\mathrm{~s}, 3 \mathrm{H} ; \mathrm{CH}_{3}\right), 1.89\left(\mathrm{~s}, 3 \mathrm{H} ; \mathrm{CH}_{3}\right)$, 2.20 (s, 3H; $\left.\mathrm{CH}_{3}\right), 7.45-7.15 \mathrm{ppm}(\mathrm{m}, 10 \mathrm{H} ; \mathrm{Ar}-\mathrm{H}) ;{ }^{13} \mathrm{C} \mathrm{NMR}$ $\left(\mathrm{CDCl}_{3}, 75 \mathrm{MHz}\right) \delta=11.1\left(\mathrm{~s} ; \mathrm{CH}_{3}\right), 13.0\left(\mathrm{~s} ; \mathrm{CH}_{3}\right), 31.0(\mathrm{~s} ;$ $\mathrm{CH}_{3}$ ), 120.8 (CC), 121.7 (CC), 126.9 (CC), 128.1 (CHar), 128.4 (CHar), 128.7 (CHar), 129.5 (CHar), 131.7 (CHar), 132.5 (CC), 135.2 (CC), 135.4 (CC), 137.4 (CC), 196.8 ppm (C=O); ESI-MS (positive mode) $m / z=323.1[\mathrm{M}]^{+}$.

1-(1-Benzyl-4-(4-chlorophenyl)-2,5-dimethyl-1H-pyrrol-3yl)ethanone (lh). Yellow solid (565 mg 67\%); ${ }^{1} \mathrm{H} \mathrm{NMR}\left(\mathrm{CDCl}_{3}\right.$, $300 \mathrm{MHz}) \delta=1.95\left(\mathrm{~s}, 3 \mathrm{H} ; \mathrm{CH}_{3}\right), 2.07\left(\mathrm{~s}, 3 \mathrm{H} ; \mathrm{CH}_{3}\right), 2.49(\mathrm{~s}, 3 \mathrm{H}$; $\mathrm{CH}_{3}$ ), 5.12 (s, 2H; $\left.\mathrm{CH}_{2}\right), 6.97-7.39$ ppm (m, 9H; $\left.\mathrm{Ar}-\mathrm{H}\right) ;{ }^{13} \mathrm{C}$ $\operatorname{NMR}\left(\mathrm{CDCl}_{3}, 75 \mathrm{MHz}\right) \delta=10.3\left(\mathrm{~s} ; \mathrm{CH}_{3}\right), 11.8\left(\mathrm{~s} ; \mathrm{CH}_{3}\right), 31,2(\mathrm{~s} ;$ $\left.\mathrm{CH}_{3}\right), 46.9\left(\mathrm{~s} ; \mathrm{CH}_{2}\right), 121.1$ (CC), 121.6 (CC), 125.6 (CHar), 126.2 (CC), 127.5 (CHar), 128.4 (CHar), 128.9 (CHar), 131.9 (CHar), 132.5 (CC), 134.5 (CC), 135.6 (CC), 136.6 (CC), 196.8 (C=O) ppm; ESI-MS (positive mode) $\mathrm{m} / z=337.1[\mathrm{M}]^{+}$.

1-(4-(4-Chlorophenyl)-2,5-dimethyl-1-(1-phenylethyl)-1Hpyrrol-3-yl)ethanone (1i). Brown sticky liquid (387 mg, 44\%); ${ }^{1} \mathrm{H}$ NMR $\left(\mathrm{CDCl}_{3}, 300 \mathrm{MHz}\right) \delta=1.88\left(\mathrm{~d}, 3 \mathrm{H} ; \mathrm{CH}_{3}\right), 1.91(\mathrm{~s}, 3 \mathrm{H}$; $\mathrm{CH}_{3}$ ), 1.98 (s, 3H; $\left.\mathrm{CH}_{3}\right), 2.24$ (s, 3H; $\left.\mathrm{CH}_{3}\right), 6.67(\mathrm{q}, 1 \mathrm{H} ; \mathrm{CH}$ ), 7.37-7.11 ppm (m, 9H; $\mathrm{Ar}-\mathrm{H}) ;{ }^{13} \mathrm{C} \mathrm{NMR}\left(\mathrm{CDCl}_{3}, 75 \mathrm{MHz}\right)$ $\delta=11,5\left(\mathrm{~s} ; \mathrm{CH}_{3}\right), 12.6\left(\mathrm{~s} ; \mathrm{CH}_{3}\right), 18.8\left(\mathrm{~s} ; \mathrm{CH}_{3}\right), 31.3\left(\mathrm{~s} ; \mathrm{CH}_{3}\right), 52.7$ (s; CH), 121.6 (CC), 121.9 (CC), 125.9 (CHar), 126.1 (CC), 127.3 (CHar), 128.3 (CHar), 128.7 (CHar), 131.9 (CHar), 132.5 (CC), 134.3 (CC), 135.8 (CC), 140.7 (CC), 197.1 ppm (C=O); ESI-MS (positive mode) $\mathrm{m} / z=351.1[\mathrm{M}]^{+}$.

1-(4-(4-Chlorophenyl)-1-isopropyl-2,5-dimethyl-1H-pyrrol-3-yl)ethan-1-one (1j). Brown sticky liquid (102,5 mg, 41\%); ${ }^{1} \mathrm{H}$ NMR $\left(\mathrm{CDCl}_{3}, 300 \mathrm{MHz}\right) \delta=1.56\left(2 \mathrm{~d}, 6 \mathrm{H} ; \mathrm{CH}_{3}\right), 2.10(\mathrm{~s}$, $\left.3 \mathrm{H} ; \mathrm{CH}_{3}\right), 2.19$ (s, 3H; $\mathrm{CH}_{3}$ ), 2.59 (s, $\left.3 \mathrm{H} ; \mathrm{CH}_{3}\right), 4.60-4.55$ (m, $1 \mathrm{H} ; \mathrm{CH}), 7.37-7.15 \mathrm{ppm}(\mathrm{m}, 4 \mathrm{H} ; \mathrm{Ar}-\mathrm{H}) ;{ }^{13} \mathrm{C}$ NMR $\left(\mathrm{CDCl}_{3}\right.$, $75 \mathrm{MHz}) \delta=11.8\left(\mathrm{~s} ; \mathrm{CH}_{3}\right), 12.6\left(\mathrm{~s} ; \mathrm{CH}_{3}\right), 21.9\left(\mathrm{~s} ; \mathrm{CH}_{3}\right), 21.9(\mathrm{~s} ;$ $\left.\mathrm{CH}_{3}\right), 31.2\left(\mathrm{~s} ; \mathrm{CH}_{3}\right), 47.5$ (s; $\left.\mathrm{CH}\right), 125.5$ (CC), 128.3 (CHar), 131.9 (CHar), 132.5 (CC), 133.6 (CC), 135.9 (CC), 197.6 ppm $\left(\mathrm{C}=\mathrm{O}\right.$ ); ESI-MS (positive mode) $\mathrm{m} / z=289.1[\mathrm{M}]^{+}$.

1-(2,5-Dimethyl-1-phenyl-4-( $p$-tolyl)-1H-pyrrol-3-yl) ethanone (1k). Brown sticky liquid (477 mg, 63\%); ${ }^{1} \mathrm{H}$ NMR $\left(\mathrm{CDCl}_{3}, 300 \mathrm{MHz}\right) \delta=1.87\left(\mathrm{~s}, 3 \mathrm{H} ; \mathrm{CH}_{3}\right), 2.00\left(\mathrm{~s}, 3 \mathrm{H} ; \mathrm{CH}_{3}\right)$, 2.31 (s, 3H; $\left.\mathrm{CH}_{3}\right), 2.40\left(\mathrm{~s}, 3 \mathrm{H} ; \mathrm{CH}_{3}\right), 7.54-7.22 \mathrm{ppm}(\mathrm{m}, 9 \mathrm{H}$; $\mathrm{Ar}-\mathrm{H}) ;{ }^{13} \mathrm{C}$ NMR $\left(\mathrm{CDCl}_{3}, 75 \mathrm{MHz}\right) \delta=11.1\left(\mathrm{~s} ; \mathrm{CH}_{3}\right), 13.0(\mathrm{~s}$; $\left.\mathrm{CH}_{3}\right), 21.2\left(\mathrm{~s} ; \mathrm{CH}_{3}\right), 30.9\left(\mathrm{~s} ; \mathrm{CH}_{3}\right), 121.9$ (CC), 122.1 (CC), 126.6 (CC), 128.2 (CHar), 128.6 (CHar), 128.9 (CHar), 129.4 (CHar), 130.4 (CHar), 133.8 (CC), 134.8 (CC), 136.1 (CC), 137.7 (CC), $197.3 \mathrm{ppm}(\mathrm{C}=\mathrm{O})$; ESI-MS (positive mode) $\mathrm{m} /$ $z=303.1[\mathrm{M}]^{+}$.

1-(1-Benzyl-2,5-dimethyl-4-( $p$-tolyl)-1H-pyrrol-3-yl) ethanone (11). Brown sticky liquid (610 mg, 77\%); ${ }^{1} \mathrm{H}$ NMR $\left(\mathrm{CDCl}_{3}, 300 \mathrm{MHz}\right) \delta=1.97\left(\mathrm{~s}, 3 \mathrm{H} ; \mathrm{CH}_{3}\right), 2.40\left(\mathrm{~s}, 3 \mathrm{H} ; \mathrm{CH}_{3}\right)$, $2.45\left(\mathrm{~s}, 3 \mathrm{H} ; \mathrm{CH}_{3}\right), 2.52\left(\mathrm{~s}, 3 \mathrm{H} ; \mathrm{CH}_{3}\right), 5.12\left(\mathrm{~s}, 2 \mathrm{H} ; \mathrm{CH}_{2}\right)$, 7.33-6.99 ppm (m, 9H; Ar-H); ${ }^{13} \mathrm{C}$ NMR $\left(\mathrm{CDCl}_{3}, 75 \mathrm{MHz}\right)$ $\delta=10.3\left(\mathrm{~s} ; \mathrm{CH}_{3}\right), 11.8\left(\mathrm{~s} ; \mathrm{CH}_{3}\right), 21.2\left(\mathrm{~s} ; \mathrm{CH}_{3}\right), 31.1\left(\mathrm{~s} ; \mathrm{CH}_{3}\right)$, 46.9 (s; $\mathrm{CH}_{2}$ ), 121.1 (CC), 121.6 (CC), 125.7 (CHar), 127.4 (CC), 128.2 (CHar), 128.9 (CHar), 130.6 (CHar), 134.1 (CC), 134.2 (CC), 136.1 (CC), 136.1 (CC), 196.8 ppm (C=O); ESIMS (positive mode) $\mathrm{m} / z=371.2[\mathrm{M}]^{+}$.
1-(2,5-Dimethyl-1-(1-phenylethyl)-4-( $p$-tolyl)-1H-pyrrol3 -yl)ethanone (1m). Brown sticky liquid (530 mg, 64\%); ${ }^{1} \mathrm{H}$ $\operatorname{NMR}\left(\mathrm{CDCl}_{3}, 300 \mathrm{MHz}\right) \delta=1.92\left(\mathrm{~d}, 3 \mathrm{H} ; \mathrm{CH}_{3}\right), 1.93(\mathrm{~s}, 3 \mathrm{H}$; $\left.\mathrm{CH}_{3}\right), 1.95$ (s, 3H; $\left.\mathrm{CH}_{3}\right), 2.40$ (s, 3H; $\left.\mathrm{CH}_{3}\right), 2.45$ (s, $3 \mathrm{H} ; \mathrm{CH}_{3}$ ), $5.62(\mathrm{q}, 1 \mathrm{H} ; \mathrm{CH}), 7.39-7.13 \mathrm{ppm}(\mathrm{m}, 9 \mathrm{H} ; \mathrm{Ar}-\mathrm{H}) ;{ }^{13} \mathrm{C} \mathrm{NMR}$ $\left(\mathrm{CDCl}_{3}, 75 \mathrm{MHz}\right) \delta=11.5\left(\mathrm{~s} ; \mathrm{CH}_{3}\right), 12.7\left(\mathrm{~s} ; \mathrm{CH}_{3}\right), 18.9(\mathrm{~s} ;$ $\left.\mathrm{CH}_{3}\right), 21.2\left(\mathrm{~s} ; \mathrm{CH}_{3}\right), 31.2\left(\mathrm{~s} ; \mathrm{CH}_{3}\right), 52.7(\mathrm{~s} ; \mathrm{CH}), 122.1(\mathrm{CC})$, 122.9 (CC), 122.9 (CC), 125.9 (CHar), 127.2 (CHar), 128.7 (CHar), 128.9 (CHar), 130.5 (CHar), 133.9 (CC), 134.2 (CC), 136.0 (CC), 141.0 (CC), 197.7 ppm (C=O); ESI-MS (positive mode) $m / z=331.2[\mathrm{M}]^{+}$.

1-(1-Benzyl-2,5-dimethyl-4-(naphthalen-1-yl)-1H-pyrrol3-yl)ethanone (1n). Brown sticky liquid (327 mg, 37\%); ${ }^{1} \mathrm{H}$ $\operatorname{NMR}\left(\mathrm{CDCl}_{3}, 300 \mathrm{MHz}\right) \delta=1.48\left(\mathrm{~s}, 3 \mathrm{H} ; \mathrm{CH}_{3}\right), 1.68(\mathrm{~s}, 3 \mathrm{H}$; $\left.\mathrm{CH}_{3}\right), 2.44\left(\mathrm{~s}, 3 \mathrm{H} ; \mathrm{CH}_{3}\right), 4.97\left(\mathrm{~s}, 2 \mathrm{H} ; \mathrm{CH}_{2}\right), 7.72-6.84 \mathrm{ppm}(\mathrm{m}$, $12 \mathrm{H} ; \mathrm{Ar}-\mathrm{H}) ;{ }^{13} \mathrm{C} \mathrm{NMR}\left(\mathrm{CDCl}_{3}, 75 \mathrm{MHz}\right) \delta=10.4\left(\mathrm{~s} ; \mathrm{CH}_{3}\right)$, $12.1\left(\mathrm{~s} ; \mathrm{CH}_{3}\right), 30.2\left(\mathrm{~s} ; \mathrm{CH}_{3}\right), 46.9\left(\mathrm{~s} ; \mathrm{CH}_{2}\right), 119.9(\mathrm{CC}), 122.3$ (CC), 125.7 (CHar), 126.2 (CHar), 126.6 (CHar), 127.0 (CHar), 128.2 (CHar), 128.7 (CHar), 129.0 (CHar), 130.7 (CHar), 127.6 (CC), 127.6 (CC), 133.8 (CC), 135.1 (CC), 135.1 (CC), 136.9 (CC), 196.7 ppm (C=O); ESI-MS (positive mode) $\mathrm{m} / z=353.1[\mathrm{M}]^{+}$.

1-(2,5-Dimethyl-4-(naphthalen-1-yl)-1-(1-phenylethyl)$1 \mathrm{H}$-pyrrol-3-yl)ethanone (1o). Brown sticky liquid $(358 \mathrm{mg}$, $39 \%) ;{ }^{1} \mathrm{H}$ NMR $\left(\mathrm{CDCl}_{3}, 300 \mathrm{MHz}\right) \delta=1.50\left(\mathrm{~s}, 3 \mathrm{H} ; \mathrm{CH}_{3}\right)$, $1.65\left(\mathrm{~s}, 3 \mathrm{H} ; \mathrm{CH}_{3}\right), 1.88\left(\mathrm{~d}, 3 \mathrm{H} ; \mathrm{CH}_{3}\right), 2.46\left(\mathrm{~s}, 3 \mathrm{H} ; \mathrm{CH}_{3}\right)$, 5.60-5.63 (q, 1H; CH), 7.06-7.75 ppm (m, $12 \mathrm{H} ; \mathrm{Ar}-\mathrm{H}) ;{ }^{13} \mathrm{C}$ NMR $\left(\mathrm{CDCl}_{3}, 75 \mathrm{MHz}\right) \delta=11.7\left(\mathrm{~s} ; \mathrm{CH}_{3}\right), 13.07\left(\mathrm{~s} ; \mathrm{CH}_{3}\right)$, $18.9\left(\mathrm{~s} ; \mathrm{CH}_{3}\right), 30.3\left(\mathrm{~s} ; \mathrm{CH}_{3}\right), 52.7(\mathrm{~s} ; \mathrm{CH}), 120.0(\mathrm{CC}), 120.1$ (CC), 123.7 (CC), 125.4 (CHar), 125.7 (CHar), 126.2 (CHar), 126.9 (CC), 127.3 (CHar), 128.2 (CHar), 128.2 (CHar), 128.7 (CHar), 133.7 (CC), 134.9 (CC), 135.2 (CC), 141.1 (CC), $197.0 \mathrm{ppm}(\mathrm{C}=\mathrm{O}$ ); ESI-MS (positive mode) $\mathrm{m} / z=367.1$ $[\mathrm{M}]^{+}$.

1-(1-Isopropyl-2,5-dimethyl-4-(naphthalen-1-yl)-1H-pyrrol-3-yl)ethanone (1p). Brown sticky liquid (686 mg, 90\%); ${ }^{1} \mathrm{H}$ NMR $\left(\mathrm{CDCl}_{3}, 300 \mathrm{MHz}\right) \delta=1.57\left(2 \mathrm{~d}, 6 \mathrm{H} ; \mathrm{CH}_{3}\right), 1.99(\mathrm{~s}, 3 \mathrm{H}$; $\left.\mathrm{CH}_{3}\right), 2.19$ (s, 3H; $\left.\mathrm{CH}_{3}\right), 2.72\left(\mathrm{~s}, 3 \mathrm{H} ; \mathrm{CH}_{3}\right), 4.73(\mathrm{~m}, 1 \mathrm{H} ; \mathrm{CH})$, 7.38-7.89 ppm (m, 7H; $\mathrm{Ar}-\mathrm{H}) ;{ }^{13} \mathrm{C} \mathrm{NMR}\left(\mathrm{CDCl}_{3}, 75 \mathrm{MHz}\right)$ $\delta=12.3\left(\mathrm{~s} ; \mathrm{CH}_{3}\right), 12.9\left(\mathrm{~s} ; \mathrm{CH}_{3}\right), 21.9\left(\mathrm{~s} ; \mathrm{CH}_{3}\right), 22.0\left(\mathrm{~s} ; \mathrm{CH}_{3}\right)$, $30.3\left(\mathrm{~s} ; \mathrm{CH}_{3}\right), 47.5(\mathrm{~s} ; \mathrm{CH}), 120.3$ (CC), 122.3 (CC), 125.7 (CHar), 126.1 (CHar), 126.3 (CC), 127.4 (CHar), 128.1 (CHar), 128.7 (CHar), 133.7 (CC), 133.7 (CC), 134.2 (CC), 135.4 (CC), $197.0 \mathrm{ppm}(\mathrm{C}=\mathrm{O})$; ESI-MS (positive mode) $\mathrm{m} / z=305.1[\mathrm{M}]^{+}$.

Ethyl 1-benzyl-2-methyl-4-( $p$-tolyl)-1H-pyrrole-3-carboxylate (1q). Brown sticky liquid (241 mg, 29\%); ${ }^{1} \mathrm{H}$ NMR $\left(\mathrm{CDCl}_{3}, 300 \mathrm{MHz}\right) \delta=1.17\left(t, 3 \mathrm{H} ; \mathrm{CH}_{3}\right), 2.11\left(\mathrm{~s}, 3 \mathrm{H} ; \mathrm{CH}_{3}\right)$, $2.31\left(\mathrm{~s}, 3 \mathrm{H} ; \mathrm{CH}_{3}\right), 3.99$ (q, 2H; $\left.\mathrm{CH}_{2}\right), 4.86\left(\mathrm{~s}, 2 \mathrm{H} ; \mathrm{CH}_{2}\right), 6.39$ (s, 1H; CH), 6.87-7.25 ppm (m, 9H; Ar-H); ${ }^{13} \mathrm{C}$ NMR $\left(\mathrm{CDCl}_{3}, 75 \mathrm{MHz}\right) \delta=11.5\left(\mathrm{~s} ; \mathrm{CH}_{3}\right), 14.2\left(\mathrm{~s} ; \mathrm{CH}_{3}\right), 21.2(\mathrm{~s} ;$ $\left.\mathrm{CH}_{3}\right), 50.4\left(\mathrm{~s} ; \mathrm{CH}_{2}\right), 59.3\left(\mathrm{~s} ; \mathrm{CH}_{2}\right), 111.0(\mathrm{CC}), 120.4(\mathrm{CHar})$, 126.2 (CC), 126.8 (CHar), 127.7 (CHar), 128.3 (CHar), 128.9 (CHar), 129.2 (CHar), 132.9 (CC), 135.5 (CC), 136.3 (CC), 136.9 (CC), $165.9 \mathrm{ppm}(\mathrm{OC}=\mathrm{O})$; ESI-MS (positive mode) $\mathrm{m} /$ $z=333.1[\mathrm{M}]^{+}$.

Ethyl 2-methyl-1,4-diphenyl-1H-pyrrole-3-carboxylate (1r). $(220 \mathrm{mg}, 43 \%) ;{ }^{1} \mathrm{H} \mathrm{NMR}\left(\mathrm{CDCl}_{3}, 300 \mathrm{MHz}\right) \delta=1.18(t$, $\left.3 \mathrm{H} ; \mathrm{CH}_{3}\right), 1.98\left(\mathrm{~s}, 3 \mathrm{H} ; \mathrm{CH}_{3}\right), 4.07\left(\mathrm{q}, 2 \mathrm{H} ; \mathrm{CH}_{2}\right), 7.05(\mathrm{~s}, 1 \mathrm{H}$; $\mathrm{CH})$, 7.04-7.37 ppm (m, $10 \mathrm{H}$; $\mathrm{Ar}-\mathrm{H}) ;{ }^{13} \mathrm{C} \mathrm{NMR}\left(\mathrm{CDCl}_{3}\right.$, 


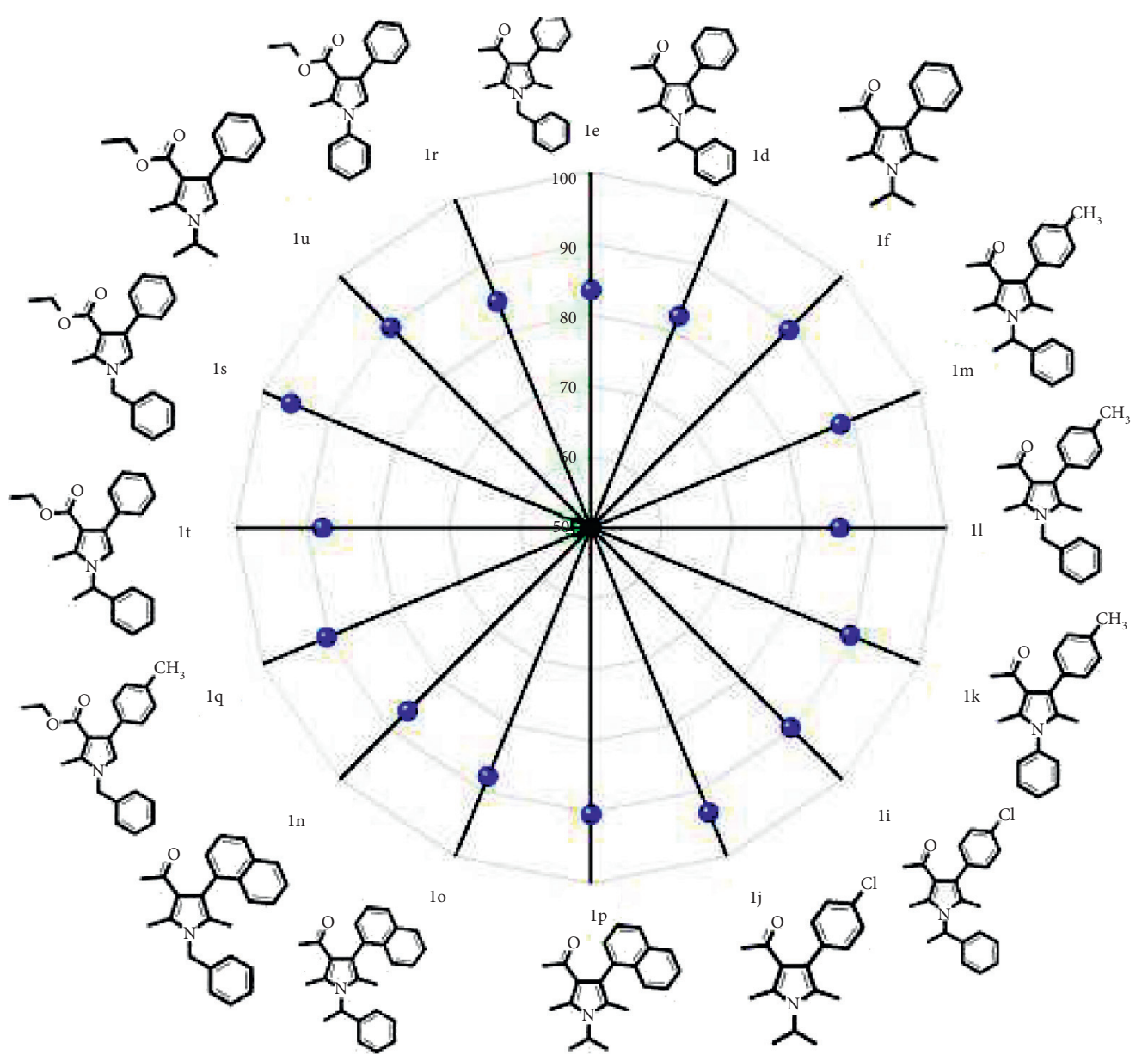

FIGURE 2: Distribution of inhibition efficiency of different compounds.

$75 \mathrm{MHz}) \delta=13.8\left(\mathrm{~s} ; \mathrm{CH}_{3}\right), 18.6\left(\mathrm{~s} ; \mathrm{CH}_{3}\right), 59.9\left(\mathrm{~s} ; \mathrm{CH}_{2}\right), 105.8$ (CC), 126.2 (CHar), 127.1-131.0 (CHar), 140.4 (CC), 147.2 (CC), $168.1 \mathrm{ppm}(\mathrm{OC}=\mathrm{O})$; ESI-MS (positive mode) $\mathrm{m} /$ $z=305.2[\mathrm{M}]^{+}$.

Ethyl 1-benzyl-2-methyl-4-phenyl-1H-pyrrole-3-carboxylate (1s). Brown sticky liquid (343 mg, 43\%); ${ }^{1} \mathrm{H}$ NMR $\left(\mathrm{CDCl}_{3}, 300 \mathrm{MHz}\right) \delta=1.24\left(t, 3 \mathrm{H} ; \mathrm{CH}_{3}\right), 2.55\left(\mathrm{~s}, 3 \mathrm{H} ; \mathrm{CH}_{3}\right)$, 4.27 (q, 2H; $\left.\mathrm{CH}_{2}\right), 5.09\left(\mathrm{~s}, 2 \mathrm{H} ; \mathrm{CH}_{2}\right), 6.66(\mathrm{~s}, 1 \mathrm{H} ; \mathrm{CH})$, 7.13-7.52 ppm (m, 10H; $\mathrm{Ar}-\mathrm{H}) ;{ }^{13} \mathrm{C} \mathrm{NMR}\left(\mathrm{CDCl}_{3}, 75 \mathrm{MHz}\right)$ $\delta=11.5\left(\mathrm{~s} ; \mathrm{CH}_{3}\right), 14.2\left(\mathrm{~s} ; \mathrm{CH}_{3}\right), 50.6\left(\mathrm{~s} ; \mathrm{CH}_{2}\right), 59.4\left(\mathrm{~s} ; \mathrm{CH}_{2}\right)$, 111.3 (CC), 120.5-129.4 (CHar), 126.2 (CC), 136.1 (CC), 136.5 (CC), 136.9 (CC), $165.9 \mathrm{ppm}$ (OC=O); ESI-MS (positive mode) $\mathrm{m} / z=319.1[\mathrm{M}]^{+}$.

Ethyl 2-methyl-4-phenyl-1-(1-phenylethyl)-1H-pyrrole-3-carboxylate (1t). Brown sticky liquid (341 mg, $41 \%) ;{ }^{1} \mathrm{H} \mathrm{NMR}\left(\mathrm{CDCl}_{3}, 300 \mathrm{MHz}\right) \delta=1.05\left(t, 3 \mathrm{H} ; \mathrm{CH}_{3}\right)$, $1.72\left(\mathrm{~d}, 3 \mathrm{H} ; \mathrm{CH}_{3}\right), 2.4\left(\mathrm{~s}, 3 \mathrm{H} ; \mathrm{CH}_{3}\right), 4.05$ (q, $2 \mathrm{H} ; \mathrm{CH}_{2}$ ), $5.28(\mathrm{q}, 1 \mathrm{H} ; \mathrm{CH}), 6.64(\mathrm{~s}, 1 \mathrm{H} ; \mathrm{CH}), 6.96-7.28 \mathrm{ppm}(\mathrm{m}$, $10 \mathrm{H} ; \mathrm{Ar}-\mathrm{H}) ;{ }^{13} \mathrm{C} \mathrm{NMR}\left(\mathrm{CDCl}_{3}, 75 \mathrm{MHz}\right) \delta=10.3(\mathrm{~s}$; $\left.\mathrm{CH}_{3}\right), 12.9\left(\mathrm{~s} ; \mathrm{CH}_{3}\right), 21.1\left(\mathrm{~s} ; \mathrm{CH}_{3}\right), 54.0(\mathrm{~s} ; \mathrm{CH}), 58.3$ (s; $\mathrm{CH}_{2}$ ), 110.9 (CHar), 115.8 (CHar), 124.4 (CHar), 125.0 (CHar), 125.1 (CHar), 126.4 (CHar), 126.0 (CHar), 127.8 (CHar), 128.2 (CHar), 135.1 (CC), 135.3 (CC), 135.7 (CC), 141.1 (CC), 165.2 ppm (OC=O); ESI-MS (positive mode) $m / z=333.2[\mathrm{M}]^{+}$.
Ethyl 1-isopropyl-2-methyl-4-phenyl-1H-pyrrole-3carboxylate (1u). Brown sticky liquid (196 mg, 29\%); ${ }^{1} \mathrm{H}$ $\mathrm{NMR}\left(\mathrm{CDCl}_{3}, 300 \mathrm{MHz}\right) \delta=1.19\left(t, 3 \mathrm{H} ; \mathrm{CH}_{3}\right), 1.38(2 \mathrm{~d}, 6 \mathrm{H}$; $\mathrm{CH}_{3}$ ), 2.48 (s, 3H; $\left.\mathrm{CH}_{3}\right), 4.07$ (q, $\left.2 \mathrm{H} ; \mathrm{CH}_{2}\right), 4.28(\mathrm{~m}, 1 \mathrm{H}$; $\mathrm{CH}), 6.56$ (s, 1H; CH), 7.14-7,47 ppm (m, 5H; $\mathrm{Ar}-\mathrm{H}) ;{ }^{13} \mathrm{C}$ $\mathrm{NMR}\left(\mathrm{CDCl}_{3}, 75 \mathrm{MHz}\right) \delta=10.1\left(\mathrm{~s} ; \mathrm{CH}_{3}\right), 13.0\left(\mathrm{~s} ; \mathrm{CH}_{3}\right), 22.2$ $\left(\mathrm{s} ; \mathrm{CH}_{3}\right), 28.7\left(\mathrm{~s} ; \mathrm{CH}_{3}\right), 46.0(\mathrm{~s} ; \mathrm{CH}), 58.2\left(\mathrm{~s} ; \mathrm{CH}_{2}\right), 110.2$ (CC), 114.1 (CHar), 124.9 (CHar), 126.9 (CC), 127.3 (CHar), 130.5 (CHar), 134.3 (CC), 135.3 (CC), 165.0 ppm (OC=O); ESI-MS (positive mode) $m / z=271.1[\mathrm{M}]^{+}$.

4-(Benzylamino)pent-3-en-2-one. Brown sticky liquid (315 mg, 98\%). ${ }^{1} \mathrm{H}$ NMR $\left(\mathrm{CDCl}_{3}, 300 \mathrm{MHz}\right) \delta=1,79$ (s, 3H; $\left.\mathrm{CH}_{3}\right), 1.89$ (s, 3H; $\left.\mathrm{CH}_{3}\right), 4.29$ (s, 2H; $\left.\mathrm{CH}_{2}\right), 8.80$ (s, $\left.1 \mathrm{H} ; \mathrm{CH}\right)$, 7.11-7.16 (m, 5H; Ar-H), 11,04 ppm (s, 1H; NH); ${ }^{13} \mathrm{C}$ NMR $\left(\mathrm{CDCl}_{3}, 75 \mathrm{MHz}\right) \delta=18.65\left(\mathrm{~s} ; \mathrm{CH}_{3}\right), 28.68\left(\mathrm{~s} ; \mathrm{CH}_{3}\right), 46.45(\mathrm{~s} ;$ $\mathrm{CH}_{2}$ ), 95.85 (s; $\left.\mathrm{C}=\mathrm{CH}\right), 126.43$ (CHar), 127.23 (CHar), 128.65 (CHar), 138.03 (CHar), 162.54 (CC), 194.70 ppm $(\mathrm{C}=\mathrm{O})$.

3.6. Electrochemical Study. Corrosion leads to a significant loss of materials, energy, and money [33]. This results in a loss of products in industrial installations and can lead to serious accidents and contribute to environmental pollution. However, there are many ways to prevent and stem this 
TABLE 5: Electrochemical parameters of steel in $1 \mathrm{M} \mathrm{HCl}$ with and without different compounds at $10^{-3} \mathrm{M}$.

\begin{tabular}{lccc}
\hline Inhibitor $\left(10^{-3} \mathrm{M}\right)$ & $\mathrm{i}_{\text {corr }}\left(\mathrm{mA} / \mathrm{cm}^{2}\right)$ & $\mathrm{E}_{\text {corr }}(\mathrm{Ag} / \mathrm{AgCl})$ & $\eta(\%)$ \\
\hline Blank & 0.682 & -379 & -- \\
$1 \mathrm{e}$ & 0.116 & -361 & 83 \\
$1 \mathrm{~d}$ & 0.123 & -363 & 82 \\
1f & 0.071 & -333 & 90 \\
$1 \mathrm{~m}$ & 0.083 & -354 & 88 \\
$1 \mathrm{l}$ & 0.105 & -383 & 85 \\
$1 \mathrm{k}$ & 0.072 & -366 & 89 \\
$1 \mathrm{i}$ & 0.072 & -366 & 89 \\
$1 \mathrm{j}$ & 0.052 & -352 & 92 \\
$1 \mathrm{p}$ & 0.067 & -375 & 90 \\
$1 \mathrm{o}$ & 0.085 & -348 & 87 \\
$1 \mathrm{n}$ & 0.094 & -383 & 86 \\
$1 \mathrm{q}$ & 0.068 & -363 & 90 \\
$1 \mathrm{t}$ & 0.085 & -372 & 87 \\
$1 \mathrm{~s}$ & 0.029 & -308 & 96 \\
$1 \mathrm{u}$ & 0.070 & -356 & 90 \\
$1 \mathrm{r}$ & 0.108 & -369 & 84 \\
\hline
\end{tabular}

phenomenon. Among them, corrosion inhibitors are used to control and reduce metal corrosion in aqueous environments $[34,35]$. Thus, we have tested the synthesized polysubstituted pyrroles as corrosion inhibitors of S300 steel in $1 \mathrm{M}$ hydrochloric acid, and the results are presented in Figure 2.

The data in Figure 2 clearly show that the addition of different pyrrolic molecules inhibits the steel corrosion. The presence of an inhibitor clearly affects the corrosion mechanism. It could be attributed to the adsorption process of active molecules onto the steel surface leading to an increase in surface coverage $[36,37]$. The adsorption phenomena could be occurring due to the donor-acceptor interactions between the vacant d-orbital of the steel surface and the lone-pair electron of the heteroatom, as well as the aromatic rings of inhibitors [38].

It can be seen from Table 5 that the inhibition efficiency of different compounds varies from $82 \%$ to $96 \%$. The results summarized in Table 5 clearly show that the maximum displacement of corrosion potential occurred around $69 \mathrm{mV}$ compared to the blank. This result reveals that different compounds act as mixed-type inhibitors [39].

\section{Conclusion}

In conclusion, we have successfully performed an environmentally friendly, operationally simple, economical, and efficient synthesis of pyrrole derivatives by a one-pot synthesis via a four-component domino reaction. This green protocol, using 1,3-dicarbonyl compounds, aromatic aldehydes, aliphatic and aromatic amines, and nitroalkanes compounds in the presence of natural hydroxyapatite in solvent-free reaction, leads to the synthesis of highly functionalized tetra- or penta-substituted pyrroles with a wide variety of substrates. The advantage of this protocol is the use of hydroxyapatite as a cheap, natural, and nontoxic catalyst. Finally, different compounds were found to be good corrosion inhibitors for
S300 steel in a $1 \mathrm{M} \mathrm{HCl}$ solution at $10^{-3} \mathrm{M}$. The maximum inhibition efficiency was found to be around $96 \%$, and the inhibitors revealed a mixed behavior.

\section{Data Availability}

No data were used to support this study.

\section{Conflicts of Interest}

The authors declare that they have no conflicts of interest.

\section{Supplementary Materials}

NMR and mass spectrum of some significant prepared products. (Supplementary Materials)

\section{References}

[1] J. Qiu, T. Liang, J. Wu et al., "N-substituted pyrrole derivative $12 \mathrm{M}$ inhibits HIV-1 entry by targeting gp41 of HIV-1 envelope glycoprotein," Frontiers in Pharmacology, vol. 10, pp. 1-12, 2019.

[2] M. C. Boukouvala, N. G. Kavallieratos, C. G. Athanassiou, G. Benelli, and L. P. Hadjiarapoglou, "Insecticidal efficacy of six new pyrrole derivatives against four stored-product pests," Environmental Science and Pollution Research, vol. 26, no. 29, pp. 29845-29856, 2019.

[3] K. M. John and A. Joule, Heterocyclic Chemistry, Wiley Online Library, Hoboken, NJ, USA, 5th edition, 2010.

[4] A. S. Plaskon, S. V. Ryabukhin, D. M. Volochnyuk, A. N. Shivanyuk, and A. A. Tolmachev, "A synthesis of 5hetaryl-3-(2-hydroxybenzoyl) pyrroles," Tetrahedron, vol. 64, no. 25, pp. 5933-5943, 2008.

[5] C.-F. Lee, L.-M. Yang, T.-Y. Hwu, A.-S. Feng, J.-C. Tseng, and T.-Y. Luh, "One-pot synthesis of substituted furans and pyrroles from propargylic dithioacetals. New annulation route to highly photoluminescent oligoaryls," Journal of the American Chemical Society, vol. 122, no. 20, pp. 4992-4993, 2000.

[6] V. Estévez, M. Villacampa, and J. C. Menéndez, "Recent advances in the synthesis of pyrroles by multicomponent reactions," Chemical Society Reviews, vol. 43, no. 13, pp. 4633-4657, 2014.

[7] H. Gao, J. Sun, and C.-G. Yan, "Synthesis of functionalized 2pyrrolidinones via domino reactions of arylamines, ethyl glyoxylate and acetylenedicarboxylates," Tetrahedron, vol. 69, no. 2, pp. 589-594, 2013.

[8] D. V. Ley, G. K. C. Nicolaou, and L. E. O. A Padwa, Synthesis of Heterocycles via Multicomponent Reactions II, Vol. 25, Springer Berlin Heidelberg, Berlin, Heidelberg, Germany, 2010.

[9] S. Maiti, S. Biswas, and U. Jana, "Iron (III)-Catalyzed fourcomponent coupling reaction of 1,3-dicarbonyl compounds, amines, aldehydes, and nitroalkanes: a simple and direct synthesis of functionalized pyrroles," The Journal of Organic Chemistry, vol. 75, no. 5, pp. 1674-1683, 2010.

[10] G. R. Reddy, T. R. Reddy, S. C. Joseph, K. S. Reddy, and M. Pal, "Iodine catalyzed four-component reaction: a straightforward one-pot synthesis of functionalized pyrroles under metal-free conditions," RSC Advances, vol. 2, no. 8, p. 3387, 2012.

[11] A. T. Khan, M. Lal, P. Ray Bagdi, R. Sidick Basha, P. Saravanan, and S. Patra, "Synthesis of tetra-substituted 
pyrroles, a potential phosphodiesterase $4 \mathrm{~B}$ inhibitor, through nickel (II) chloride hexahydrate catalyzed one-pot fourcomponent reaction," Tetrahedron Letters, vol. 53, no. 32, pp. 4145-4150, 2012.

[12] H. M. Meshram, B. Madhu Babu, G. Santosh Kumar, P. B. Thakur, and V. M. Bangade, "Catalyst-free four-component protocol for the synthesis of substituted pyrroles under reusable reaction media," Tetrahedron Letters, vol. 54, no. 19, pp. 2296-2302, 2013.

[13] A. B. Atar and Y. T. Jeong, "Heterogenized tungsten complex: an efficient and high yielding catalyst for the synthesis of structurally diverse tetra substituted pyrrole derivatives via four-component assembly," Tetrahedron Letters, vol. 54, no. 41, pp. 5624-5628, 2013.

[14] S. Horikoshi, T. Hamamura, M. Kajitani, M. Yoshizawa-Fujita, and N. Serpone, "Green chemistry with a novel $5.8-\mathrm{GHz}$ microwave apparatus. Prompt one-pot solvent-free synthesis of a major ionic liquid: the 1-Butyl-3-methylimidazolium tetrafluoroborate system," Organic Process Research \& Development, vol. 12, no. 6, pp. 1089-1093, 2008.

[15] K. R. Seddon, A. Stark, and M.-J. Torres, "Influence of chloride, water, and organic solvents on the physical properties of ionic liquids," Pure and Applied Chemistry, vol. 72, no. 12, pp. 2275-2287, 2000.

[16] B. Jastorff, K. Mölter, P. Behrend et al., "Progress in evaluation of risk potential of ionic liquids-basis for an eco-design of sustainable products," Green Chemistry, vol. 7, no. 5, p. 362, 2005.

[17] A. L. Gajengi, C. S. Fernandes, and B. M. Bhanage, "Synthesis of $\mathrm{Cu} 2 \mathrm{O} / \mathrm{Ag}$ nanocomposite and their catalytic application for the one pot synthesis of substituted pyrroles," Molecular Catalysis, vol. 451, pp. 13-19, 2018.

[18] A. L. Gajengi and B. M. Bhanage, "NiO nanoparticles: efficient catalyst for four component coupling reaction for synthesis of substituted pyrroles," Catalysis Letters, vol. 146, no. 8, pp. 1341-1347, 2016.

[19] L. A. Pachechne, V. F. Pereira, G. M. Martins, E. Martendal, F. R. Xavier, and S. R. Mendes, "One-pot multicomponent synthesis of 1,2,3,4-tetrasubstituted pyrroles catalyzed by [NMPH]CH3SO3," Tetrahedron Letters, vol. 60, no. 38, p. 151043, 2019.

[20] M. Hassani, M. R. Naimi-Jamal, and L. Panahi, "One-pot multicomponent synthesis of substituted pyrroles by using chitosan as an organocatalyst," ChemistrySelect, vol. 3, no. 2, pp. 666-672, 2018.

[21] R. K. Gupta, M. Malviya, C. Verma, N. K. Gupta, and M. A. Quraishi, "Pyridine-based functionalized graphene oxides as a new class of corrosion inhibitors for mild steel: an experimental and DFT approach," RSC Advances, vol. 7, no. 62 , pp. 39063-39074, 2017.

[22] J. Haque, V. Srivastava, C. Verma, and M. A. Quraishi, "Experimental and quantum chemical analysis of 2-amino-3-((4-((S)-2amino-2-carboxyethyl)-1H-imidazol-2-yl) thio) propionic acid as new and green corrosion inhibitor for mild steel in $1 \mathrm{M}$ hydrochloric acid solution," Journal of Molecular Liquids, vol. 225, pp. 848-855, 2017.

[23] C. Verma, L. O. Olasunkanmi, E. E. Ebenso, and M. A. Quraishi, "Substituents effect on corrosion inhibition performance of organic compounds in aggressive ionic solutions: a review," Journal of Molecular Liquids, vol. 251, pp. 100-118, 2018.

[24] R. Solmaz, "Investigation of the inhibition effect of 5-((E)-4phenylbuta-1,3-dienylideneamino)-1,3,4-thiadiazole-2-thiol
Schiff base on mild steel corrosion in hydrochloric acid," Corrosion Science, vol. 52, no. 10, pp. 3321-3330, 2010.

[25] A. Louroubi, A. Nayad, A. Hasnaoui et al., "Synthesis, structural characterization, theoretical studies and corrosion inhibition of a new pyrrole derivative:1-(1-benzyl-4-(4-chlorophenyl)-2,5-dimethyl-1H-pyrrol-3-yl) ethanone," Journal of Molecular Structure, vol. 1189, pp. 240-248, 2019.

[26] A. Louroubi, H. Ouahine, B. Boualy, M. A. Ali, and E. Firdoussi, "Natural hydroxyapatite $\mathrm{Ca} 5$ (PO 4) $3 \mathrm{OH}$ : an efficient catalyst for the chemo- and regioselective enamination of the $\beta$ - ketoesters," Journal of Advanced Catalysis Science and Technology, vol. 3, no. 2, pp. 63-67, 2016.

[27] S. Bose and S. K. Saha, "Synthesis and characterization of hydroxyapatite nanopowders by emulsion technique," Chemistry of Materials, vol. 15, no. 23, pp. 4464-4469, 2003.

[28] N. Phonthammachai, J. Kim, and T. J. White, "Synthesis and performance of a photocatalytic titania-hydroxyapatite composite," Journal of Materials Research, vol. 23, no. 9, pp. 2398-2405, 2008.

[29] R. Atir, S. Mallouk, K. Bougrin, M. Soufiaoui, and A. Laghzizil, "Porous calcium hydroxyapatite as an efficient catalyst for synthesis of pyrazolines via 1,3-dipolar cycoloaddition under solvent-free microwave irradiation," Synthetic Communications, vol. 36, no. 1, pp. 111-120, 2006.

[30] A. Louroubi, A. Hasnaoui, Y. A. Aicha et al., "3-Acetyl-2,5-dimethyl-1,4-diphenylpyrrole: synthesis, X-ray structure, DFT, TDDFT studies and anti-corrosion activity," Chemical Data Collection, vol. 32, Article ID 100662, 2021.

[31] A. Louroubi, R. Outouch, M. Ait Ali, A. Spannenberg, and L. El Firdoussi, "1-(1-Benzyl-2,5-dimethyl-4-phenyl-1H-pyrrol-3-yl) ethanone," IUCrData, vol. 2, no. 6, Article ID x170895, 2017.

[32] C. A. Grob and K. Camenish, "Eine neue pyrrolring-synthese," Helvetica Chimica Acta, vol. 36, no. 1, pp. 49-58, 1953.

[33] E. During, Corrosion Atlas 3rd Edition A Collection of Illustrated Case Histories, Elsevier, Amsterdam, Netherlands, 2018.

[34] R. Idouhli, A. Oukhrib, Y. Koumya, A. Abouelfida, A. Benyaich, and A. Benharref, "Inhibitory effect of Atlas cedar essential oil on the corrosion of steel in $1 \mathrm{~m} \mathrm{HCl}$," Corrosion Reviews, vol. 36, no. 4, pp. 373-384, Jul. 2018.

[35] L. O. Olasunkanmi, B. P. Moloto, I. B. Obot, and E. E. Ebenso, "Anticorrosion studies of some hydantoin derivatives for mild steel in $0.5 \mathrm{M} \mathrm{HCl}$ solution: experimental, quantum chemical, Monte Carlo simulations and QSAR studies," Journal of Molecular Liquids, vol. 252, pp. 62-74, 2018.

[36] R. Idouhli, A. N'Ait Ousidi, Y. Koumya et al., "Electrochemical studies of monoterpenic thiosemicarbazones as corrosion inhibitor for steel in $1 \mathrm{M} \mathrm{HCl}$," International Journal of Corrosion, vol. 2018, pp. 1-15, 2018.

[37] N. O. Eddy, P. O. Ameh, I. J. Gwarzo, I. J. Okop, and S. N. Dodo, "Physicochemical study and corrosion inhibition potential of Ficus tricopoda for aluminium in acidic medium," Portugaliae Electrochimica Acta, vol. 31, no. 2, pp. 79-93, 2013.

[38] M. Yadav, T. K. Sarkar, and I. B. Obot, "Withdrawn: indolines as novel corrosion inhibitors: electrochemical, XPS, DFT and molecular dynamics simulation studies," Corrosion Science, vol. 2017, 2017.

[39] G. A. Swetha, H. P. Sachin, A. M. Guruprasad, B. M. Prasanna, and K. H. Sudheer Kumar, "Use of seroquel as an effective corrosion inhibitor for low carbon steel in $1 \mathrm{M} \mathrm{HCl,"} \mathrm{Journal}$ of Bio- and Tribo-Corrosion, vol. 4, no. 4, p. 57, 2018. 